\title{
Identification Scheme for the Nonlinear Model of an Electro-Hydraulic Actuator
}

\author{
W.C. Leite Filho ${ }^{1} \quad$ J. Guimaraes ${ }^{2}$ \\ ${ }^{1}$ Space Mechanics and Control Division, Instituto Nacional de Pesquisas Espaciais, Brazil, waldclf@gmail.com \\ ${ }^{2}$ Space Mechanics and Control Division, Instituto Nacional de Pesquisas Espaciais, Brazil, \\ julia.guimaraes@inpe.br
}

\begin{abstract}
This work presents the building of a nonlinear model of an electro-hydraulic actuator in order to understand the limit cycle phenomenon that appears when it is used in a closed loop control system. Previously, a first harmonic analysis had been used to identify that system, but the results were unsatisfactory. Therefore, this work aims to build on that model with the use of Fast Fourier Transforms as a way to recognize previously unseen nonlinearities. Hardware in the loop tests are then used in order to find the proper parameters that create a particular limit cycle. Simulation results show that such approach is successful.
\end{abstract}

Keywords: nonlinear model, FFT, hardware in the loop, actuator model

\section{Introduction}

As part of the design of control systems of space vehicles, it is important to achieve a thorough understanding of each element modeled so that the simulated results will correctly represent the real scenario.

In particular, it is important to be able to reproduce the effect that nonlinearities create on the final output of the system, since strategies used to deal with bending modes affect the limit cycle generated by those nonlinearities.

In order to support such development, hardware in the loop (HWIL) simulations were used in an attempt to identify a proper model for the actuator, but the model proposed at the time was incomplete (Bueno and Leite Filho, 2003). A similar approach is used now in order to obtain initial values for the nonlinearities, while analysis of the Fast Fourier Transform of the signal is used to infer the missing elements.

\section{Initial Configuration}

For the initial analysis, the model proposed for the actuator has a similar configuration as the one presented in (Bueno and Leite Filho, 2003). However, further analysis of the step response indicates a slightly different third order linear model, given by the transfer function in Equation (1).

$$
T F=\frac{305500}{s^{3}+202.1 s^{2}+14520 s+326800}
$$

Hence, the model becomes the one represented in Figure 1.

The HWIL simulation used for the limit cycle analysis consisted of a simplified dynamics model of the system followed by a PD controller (Bueno and Leite Filho, 2003), as seen in Figure 2. Since both the deadzone and the backlash have known descriptive functions (Slotine and Li, 1991; Gelb and Vander Velde, 1968), the first harmonic analysis can be used to calculate the parameter values for those nonlinearities. Figure 3 shows the HWIL output for a given combination of $\mathrm{K}_{\mathrm{p}}$, $\mathrm{K}_{\mathrm{d}}$ and $\mu_{\mathrm{b}}$.

Considering $\mathrm{K}_{\mathrm{p}}=5.84, \mathrm{~K}_{\mathrm{d}}=0.062$ and $\mu_{\mathrm{b}}=12.3$ as the controller parameters, and assuming a time delay of $\mathrm{T}_{\mathrm{d}}=0.0056$, one finds $\mathrm{f}=1.1625 \mathrm{e}-04$ for the backlash and $\delta=0.0092$ for the dead-zone.

Those results correctly represent the limit cycle in both frequency and amplitude. However, as described by (Bueno and Leite Filho, 2003), this model has been unable to reproduce the shape of the signal encountered on the hardware-in-the-loop tests.

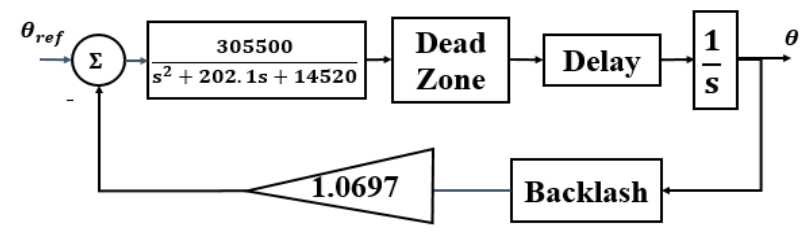

Figure 1. Initial configuration for actuator model.

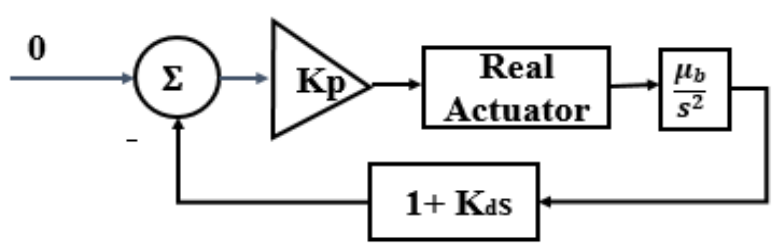

Figure 2. HWIL configuration for limit cycle analysis. 


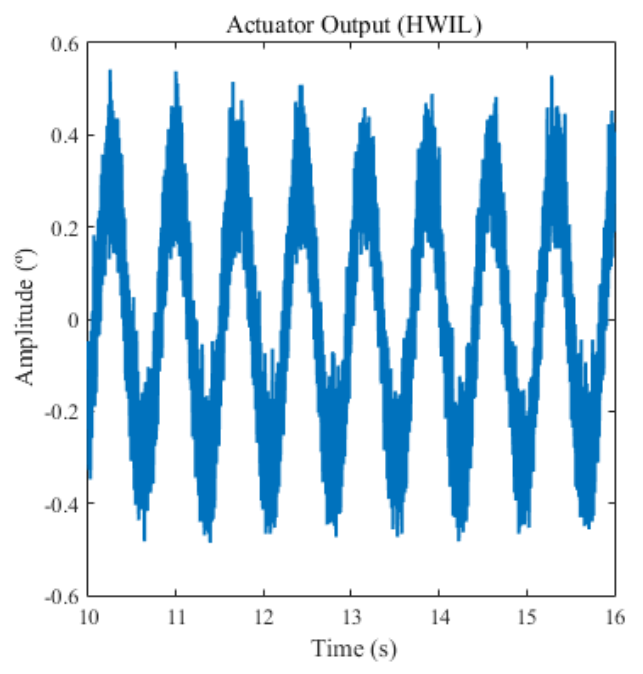

Figure 3. Actuator output (HWIL) for $\mathrm{K}_{\mathrm{p}}=5.84, \mathrm{~K}_{\mathrm{d}}=0.062$ and $\mu_{\mathrm{b}}=12.3$.

\section{FFT Analysis}

It is possible to reconstruct a signal using a finite Fourier series. This can be done by using the discrete Fourier transform (DFT) - an interpolating method capable of calculating the unknown coefficients for the series given a finite sample (Chu, 2008).

However, calculating the DFT directly is generally a procedure of order $\mathrm{N}^{2}$ and it is not advisable (Chu, 2008). Instead, the most common approach is to use the Fast Fourier Transform (FFT), an algorithm capable of calculating the DFT of a sample of complex $\mathrm{N}$ data points with a speed proportional to $\mathrm{Nlog}_{2} \mathrm{~N}$ (Cooley et al, 1967; Van Loan, 1992).

In order to obtain a more accurate look at the phenomenon studied, this work uses the Fast Fourier Transform (FFT) of the simulation output signal to better understand its properties. Once the frequency range of interest is identified, the Inverse Fast Fourier Transform (IFFT) can be used to reconstruct the signal without the influence of higher frequency noise.

The FFT analysis of the signal generated by the HWIL simulation has shown that frequencies above $10 \mathrm{~Hz}$ could be ignored. An IFFT was then created so that the shape of the actuator output could be studied without the influence of external noise, as shown by Figure 4.

Figure 4 shows that the actual output presents periodic changes in its shape around the wave's antinodes, something that was not reproduced by the previous model. This indicates the existence of a relevant nonlinear phenomenon occurring when the actuator output changes direction of motion.

The physical model of the actuator guarantees the existence of an integral on the model, as shown on Figure 1 (Moreira and Leite Filho, 1988; Gibson, 1963). Therefore, it is reasonable to assume that this occurs when the derivative of the output crosses zero.

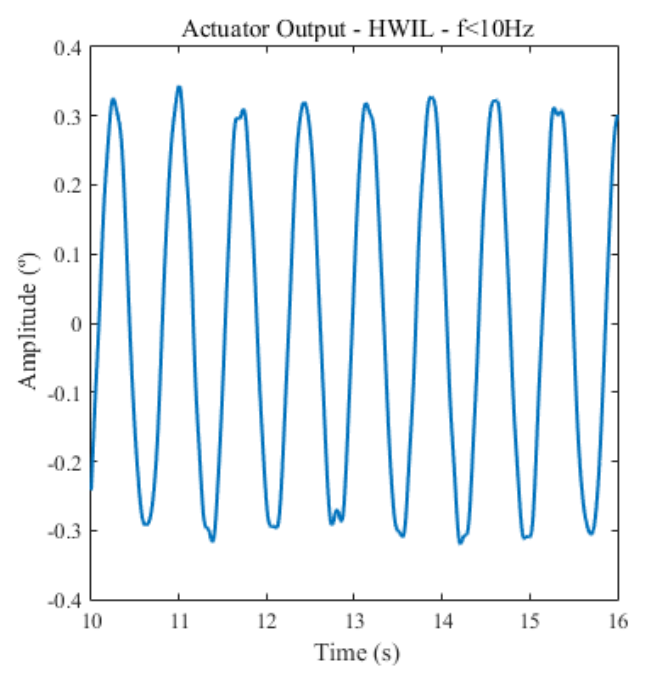

Figure 4. Actuator output (HWIL) with $\mathrm{f}<10 \mathrm{~Hz}$.

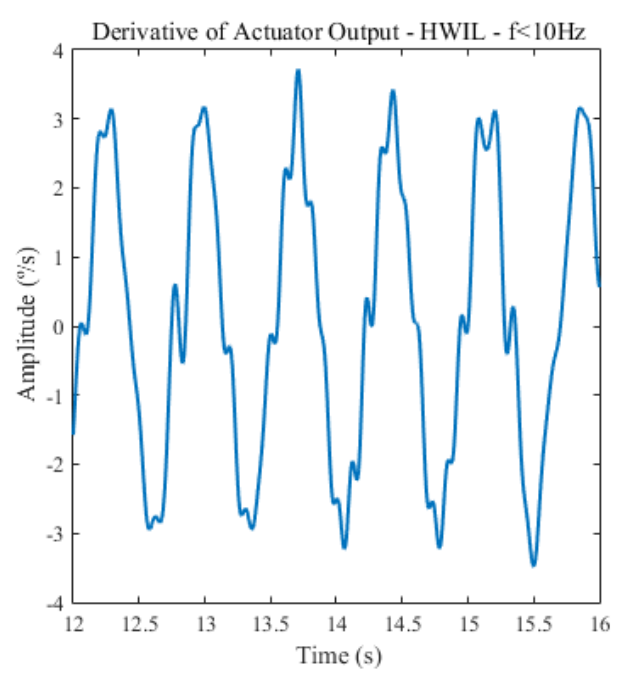

Figure 5. Reconstructed derivative.

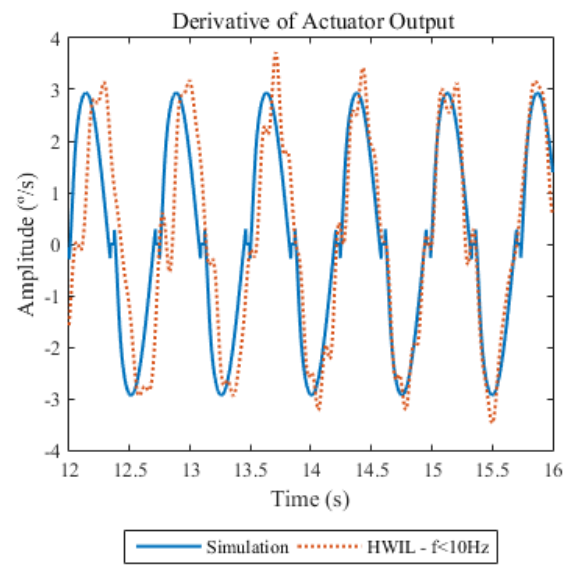

Figure 6. Derivative of actuator output including coulomb friction.

Based on the IFFT generated for frequencies smaller than $10 \mathrm{~Hz}$, as presented on Figure 4 and assuming that as the actual actuator output, it is possible to reconstruct the signal before the integral block, as shown on Figure 
5. This strategy emphasizes the nonlinearities that one wants to model. If this signal can be reproduced by the inclusion of new nonlinearities, the new model will be able to recreate the real tests.

The spikes around zero on Figure 5 indicate the existence of an offset which sign opposes the direction of the derivative. This phenomenon can be reproduced with the inclusion of a new nonlinearity, modeled as a negative Coulomb friction, before the integral block. Figure 6 shows the effect of this element on the derivative signal simulated for a given set of parameters.

Since the simulated results are still not able to reproduce completely the HWIL signal, further analysis of the derivative is necessary. In order to better understand the relevant frequencies acting on the derivative signal, the Fast Fourier Transform can be used.

Figure 7 shows a graphic of the absolute value of the FFT result with respect to frequency. This graph shows that, while the simulated model presented proportional attenuation of the higher harmonics, the real actuator showed an increase in amplitude for frequencies between $9 \mathrm{~Hz}$ and $10 \mathrm{~Hz}$, especially around the seventh harmonic $(9.57 \mathrm{~Hz})$.

Therefore, it is important to be able to represent this phenomenon in order to reproduce the real results. The presence of higher harmonics seems to indicate that those were being stimulated somewhere on the actuator. As a way to recreate this on the model, a feedback loop is proposed.

The feedback loop must be able to affect only that specific frequency band, which must be amplified somewhere on the closed loop. Thus, a feedback loop with a bandpass filter is included on the model with an appropriate gain so that the results would match the HWIL tests.

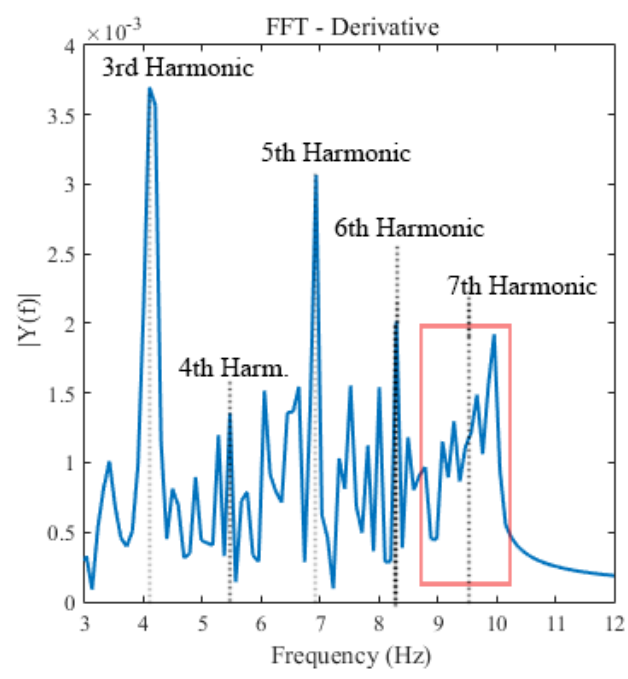

Figure 7. Absolute value of FFT - derivative of HWIL output $(\mathrm{f}<10 \mathrm{~Hz})$
The presence of the feedback loop, however, influences the step response of the model, creating an oscillating signal that does not exist in reality. As a way to attenuate this, a saturation block is added so that the feedback loop will not falsely stimulate the system when given a nonzero input.

\section{Model Structure}

The final model proposed is shown on Figure 8. The filter bandpass used was a 4th order Butterworth design, with frequencies between 9 and $10 \mathrm{~Hz}$.

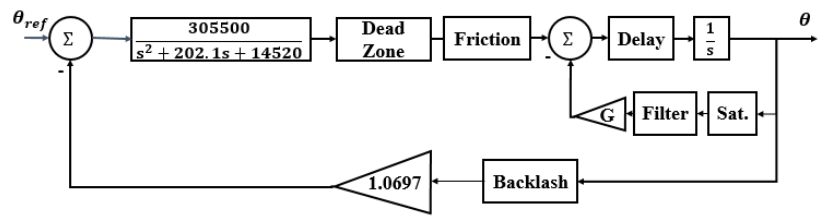

Figure 8. Final configuration for actuator model.

The presence of nonlinearities involving energy storage, such as friction, requires the use of a numerical approach in order to find the describing function (Duarte and Tenreiro Machado, 2006). Therefore, an analytical analysis no longer can be used to find the parameters that would recreate the limit cycle.

However, once a proper structure is found, different parameter values can be simulated until the response matches the HWIL results.

\section{Simulation Results}

For $K_{p}=5.84, K_{d}=0.062$ and $\mu_{b}=12.3$, the actuator parameters were tuned so that $\mathrm{f}=1.1625 \mathrm{e}-04, \delta=0.0083$, $\mathrm{T}_{\mathrm{d}}=0.0017$, offset $=-0.0054, \mathrm{G}=48$ and sat $=0.015$.

\subsection{Limit Cycle Analysis}

Initially, the model was validated by simulating the limit cycle under a PD controller in a similar configuration as described by Figure 2. The simulation results were compared to the HWIL results, analyzing both the output signal, its derivative and, finally, the absolute value of its FFT result with respect to frequency.

Figure 9 represents the derivative for both the HWIL signal and the simulation results, while Figure 10 illustrates the actuator output in both cases. Figure 11 and Figure 12 show, respectively, the absolute value Fast Fourier Transform of the derivative and the output for both the HWIL tests and the simulation results.

These results show that the new model is indeed able to recreate the limit cycle desired in frequency, amplitude and shape, showing an improvement when compared to the previous model.

However, there appears to be a periodic shift in phase that was not accounted for in this work.

Finally, since there might be more than one set of parameters that would reproduce the same limit cycle, further validation is required. 
Thus, the final configuration is used in simulations with different values of $K_{p}, K_{d}$ and $\mu_{b}$, in order to verify if those are able to recreate the HWIL results. Figure 13 exemplifies the actuator output for one of those simulations.

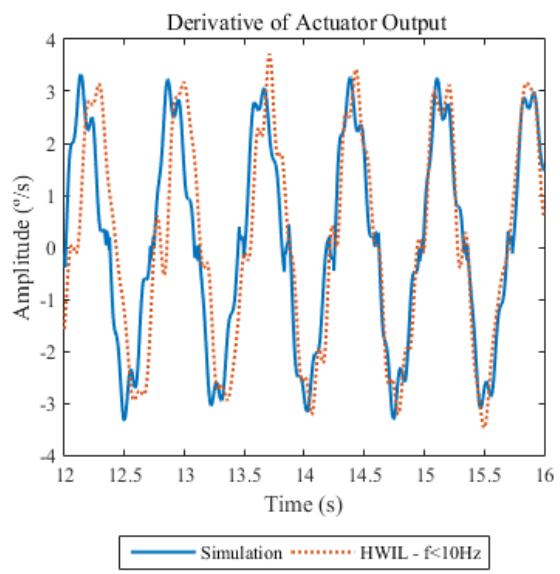

Figure 9. Derivative of actuator output for final configuration.

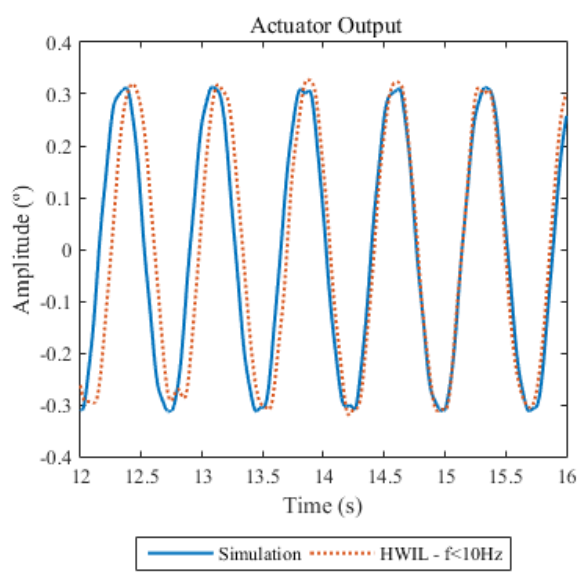

Figure 10. Actuator output for final configuration.

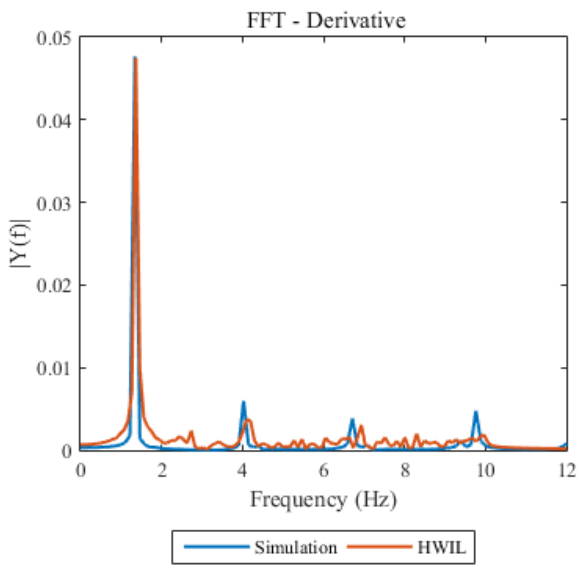

Figure 11. Absolute value of FFT of derivative of actuator output.

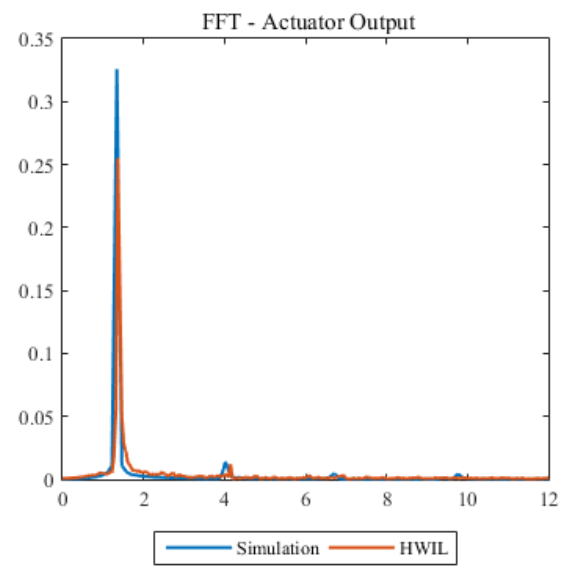

Figure 12. Absolute value of FFT of actuator output.

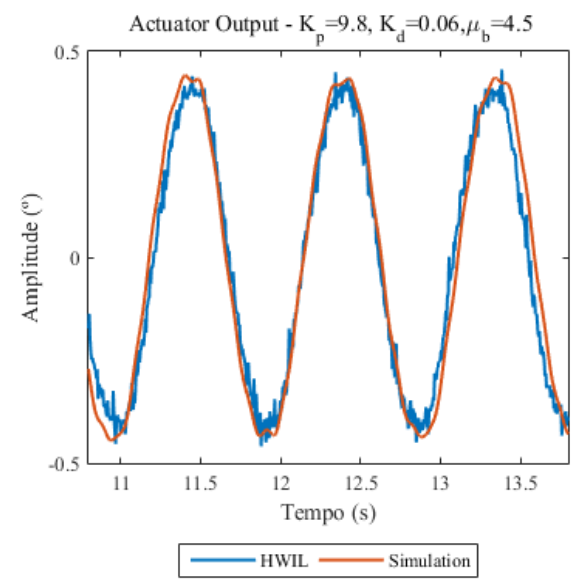

Figure 13. Actuator output for $\mathrm{K}_{\mathrm{p}}=9.8, \mathrm{~K}_{\mathrm{d}}=0.06$ and $\mu_{\mathrm{b}}=4.5$.

\subsection{Input Response}

As a way to validate the model outside of the limit cycle conditions, different inputs were simulated and the outputs compared to results from tests on a real actuator.

Simulations were made for both square and sine wave inputs. The results are shown, respectively, on Figure 14 and Figure 15, respectively.

Since the delay block was assumed to be positioned before the integral block and, therefore, inside the closed loop, the values of $T_{d}$ affected the shape of the output for a given square wave input. Thus, the acceptable values of transport delay are limited, which is why the final value used $\left(T_{d}=0.0017\right)$ is smaller than the value considered for the initial model $\left(\mathrm{T}_{\mathrm{d}}=0.0056\right)$.

As seen on Figure 15, this limiting factor has consequences on the output for the sinusoidal wave, where the actual actuator presents a higher delay than this model can reproduce.

A possible solution for this problem is to move the time delay block to after the feedback loop. This would solve the issue regarding the shape of the response to a square wave input and allow larger values for $\mathrm{Td}$. 


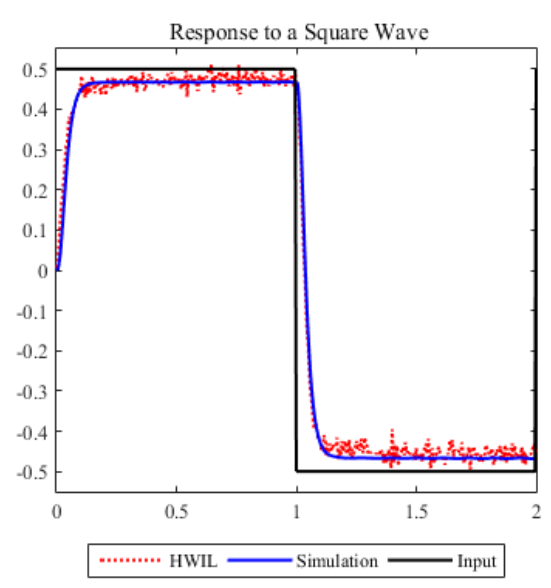

Figure 14. Actuator response for a square input.

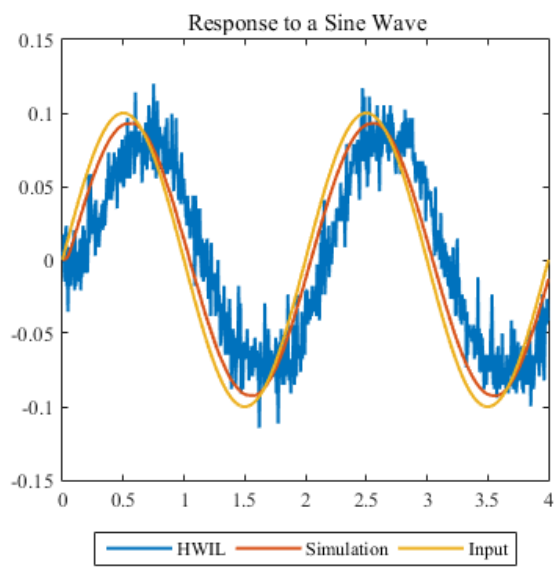

Figure 15. Actuator response for a sinusoidal input.

However, when this was implemented, no combination of parameters could be found where the results would be reproduced for all the available sets of $K_{d}, K_{p}$ and $\mu_{b}$. In most cases, when the results could be reproduced for a given set of controller parameters, the output for a different set would either generate wrong amplitudes or create system instability.

\section{Conclusions}

This paper presents a successful scheme of inferring a possible nonlinear configuration of a model based on the analysis of the FFT response of a reference signal and a complete simulation of the limit cycle. The validation of the parameters chosen for the actuator model is made by checking how the model's limit cycle responded to several HWIL parameters, as well as different inputs.

This study is based on data from a real actuator used for thrust vector control as part of the Brazilian Satellite Launcher (VLS) (Leite Filho, 1999; Leite Filho and Bueno, 2003), and the model created can be used to improve its control algorithms.
The new model was able to reproduce the HWIL results in both amplitude, frequency and shape, unlike the previous model proposed in (Bueno and Leite Filho, 2003). However, it is important to note that there seems to be a periodic phase shift along the output that was not completely reproduced by the simulation results. It is possible that this occurs because of a misplacement of the time delay block, but the model, as it stands, can be used for control systems simulations, per the original goal.

\section{References}

A. M. Bueno and W. C. Leite Filho. Parameter identification of actuator nonlinear model based on limit-cycle phenomenon. Proc. 17th Int. Congr. Mechanical Engineering. 2003.

Eleanor Chu. Discrete and Continuous Fourier Transforms: Analysis, Applications and Fast Algorithms. CRC Press. 2008.

James W. Cooley, Peter A. W. Lewis and Peter D. Welsh. Historical notes on the fast Fourier transform. IEEE Transactions on Audio and Electroacoustics, 15(2):76-79, 1967.

Fernando B. M. Duarte and J. A. Tenreiro Machado. Fractional dynamics in the describing function analysis of nonlinear friction. Proc. 2nd IFAC Workshop Fractional Differentiation and its Applications. IFAC Proceedings Volumes, 39(11):218-223, 2006.

Arthur Gelb and Wallace E. Vander Velde. Multiple-Input Describing Functions and Nonlinear System Design. McGraw-Hill. 1968.

J. E. Gibson. Nonlinear Automatic Control. McGraw-Hill, 1963.

W. C. Leite Filho. Control system of Brazilian launcher. Proc. of 4th ESA Inter. Conf. on Guidance, Navigation and Control Systems. pp. 401-405, 1999.

W. C. Leite Filho and A. M. Bueno. Analysis of limit-cycle phenomenon caused by actuator's non linearity. Proc. of 12th IASTED Inter. Conf. on Applied Simulation \& Modeling. pp. 484-489, 2003.

F. J. O. Moreira and W. C. Leite Filho. Identificação de um servomecanismo de uma tubeira móvel. Ann. VII Congresso Brasileiro de Automática. pp. 383-387, 1988.

J. Slotine and W. Li. Applied Nonlinear Control. PrenticeHall. 1991.

Charles Van Loan. Computational Frameworks for the Fast Fourier Transform. SIAM. 1992. 